\title{
miR-155-5p is Negatively Associated with Acute Pancreatitis and Inversely Regulates Pancreatic Acinar Cell Progression by Targeting Rela and Traf3
}

\author{
Sulai Liu Honglian Zou $^{\mathrm{b}}$ Yonggang Wang ${ }^{\mathrm{a}}$ Xiaohui Duan ${ }^{\mathrm{a}} \quad$ Chen Chen $^{\mathrm{a}}$ \\ Wei Cheng $^{\mathrm{a}}$ Le Wang ${ }^{\mathrm{a}}$ Ning Ning ${ }^{\mathrm{a}}$ Hongying Tang ${ }^{\mathrm{a}}$ Meifu Chen ${ }^{\mathrm{a}}$ \\ Xianhai Mao ${ }^{a}$ Chuang Peng ${ }^{a}$ Hao Lia ${ }^{a}$ Yu Jiang ${ }^{b}$ Bo Jiang ${ }^{a}$ \\ aHunan Research Center of Biliary Disease/Department of Hepatobiliary Surgery, Hunan Provincial \\ People's Hospital/The First Affiliated Hospital of Hunan Normal University, Changsha, bHunan \\ Provincial Institute of Emergency Medicine, Emergency and Critical Care Metabolomics key lab of \\ hunan province, Hunan Provincial People's Hospital/The First Affiliated Hospital of Hunan Normal \\ University, Changsha, China
}

\section{Key Words}

miR-155-5P • Acute pancreatitis $\cdot$ Rela $\bullet$ Traf3 $\bullet$ MAP $\cdot$ SAP

\begin{abstract}
Background/Aims: Acute pancreatitis contributes to high mortality in pancreatitis patients, and miRNAs play a vital role in the development of acute pancreatitis (AP), however, its precise biological role remains largely elusive. Methods: To clarify the potential mechanisms of miRNAs in AP, we built mouse models of mild acute pancreatitis (MAP) and moderate/ severe acute pancreatitis (SAP). MiRNA microarray analysis and Real-time quantitative PCR (qRT-PCR) were used to analyze the expression of miRNA in MAP/SAP. TargetScan software, dual-luciferase gene reporter assays and Western blotting were used to assess the target genes of miR-155-5p in AP. Results: miR-155-5p was significantly decreased in MAP/SAP mice compared to controls. In pancreatic acinar AR42J cells transfected with miR-155-5p mimic, the expression of Rela and Traf3 notably decreased in both the caerulein- and TLC-Sinduced groups compared with the negative control (NC); however, the expression of Rela and Traf3 notably increased after transfection with miR-155-5p inhibitor. Combined analysis using the TargetScan software and dual-luciferase gene reporter assays indicated that Rela and Traf3 were both targeted by miR-155-5p. Meanwhile, the expression of Ptgs 2 also decreased after transfection of the AR42J cells with miR-155-5p mimic. The opposite results were found when miR-155-5p inhibitor was transfected into the AR42J cells. In addition, we treated caerulein- and TLC-S-induced AR42J cells with the Rela inhibitor helenalin and found that the

\footnotetext{
Chuang Peng, Hao Li, Hunan Research Center of Biliary Disease/Dept. of Hepatobiliary Surgery, Hunan Provincial People's Hosp.

E-Mail pengchuangcn@163.com; lihao@hunnu.edu.cn; jiangyu@hunnu.edu.cn; hepatojiangbo@163.com
} Yu Jiang and Bo Jiang /The First Aff. Hosp. of Hunan Normal Univ. Changsha, Hunan (China); Tel. 08673183929520
\end{abstract}




\section{Cellular Physiology Cell Physiol Biochem 2018;51:1584-1599

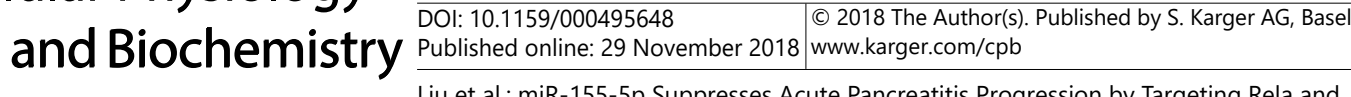 \\ Traf3}

expression of Rela, Traf3 and Ptgs2 decreased compared with the NC, while the expression of miR-155-5p did not show any significant difference. Furthermore, we found that miR-155-5p was significantly down-regulated in pancreatitis patients. Conclusion: miR-155-5p inversely regulated AP development through the Rela/Traf3/Ptgs2 signaling pathway.

(C) 2018 The Author(s)

Published by S. Karger AG, Basel

\section{Introduction}

Acute pancreatitis (AP) is an inflammatory process of the pancreas and has a broad spectrum of clinical manifestations and variations of various biochemical indexes [1]. It has been reported that the average incidence rate of AP is 19 cases per 100, 000 people per year [2]. Notably, the morbidity and mortality of severe acute pancreatitis (SAP) can reach $25-45 \%$ [3]. Considering the negative impact of AP on human health, it is urgent for us to clarify the mechanisms of occurrence and development of AP and investigate its potential therapeutic targets.

MicroRNAs are a class of 21-23 nucleotide long, non-coding, single-stranded, endogenous small RNA molecules that regulate gene expression by binding to the 3'-untranslated region (3'-UTR) of an mRNA target to promote mRNA degradation and/or translational repression $[4,5]$. It has been reported that miRNAs exert vital roles in a variety of inflammatory diseases, such as acute kidney injury [6], adipose tissue inflammation [7], inflammatory bowel disease [8], periapical lesions and human periodontal ligament fibroblast inflammation [9] and allergy and asthma [10]. In addition, a few studies have focused on the relationship between miRNAs and AP. Zhu $\mathrm{H}$ et al. found that miR-141 targeted HMGB1 in the regulation of autophagy in L-arginine-induced acute pancreatitis [11], and Gao B et al. found that the down-regulation of miR-148b-3p was associated with starvation-induced autophagy in the AR42J pancreatic acinar cell line [12]. Kuśnierz-Cabala B et al. demonstrated that the expression levels of miR-126-5p, miR-148a-3p, miR-216a-5p, miR-551b-5p and miR-375 were significantly elevated in moderate and severe acute pancreatitis (SAP) when compared to control subjects; however, in mild acute pancreatitis (MAP) patients, only miR-216a-5p, miR-551b-5p, and miR-375 were highly expressed [13]. Ma X et al. reported that miR-21 was overexpressed in a murine model of AP [14], and An F et al. discovered that miR-24$3 p$, miR-361-5p, miR-1246 and miR-222-3p were constantly up-regulated, and miR-181a-5p was constantly down-regulated, in hypertriglyceridemia-induced acute pancreatitis patients [15]. Moreover, it has been reported that miR-375, miR-217, miR-148a, miR-216a, miR-122, miR-214 and miR-138 were increased in mesenteric lymph nodes from rats with AP, and miR217, miR-375, miR-122 and miR-148a were also increased in matched rat plasma samples [16]. Though many miRNAs have been reported as biomarkers for AP, additional research is necessary regarding miR-155-5p, which is involved in chronic inflammation caused by adipose tissue [17], and whose role in the development of AP is still not clear.

In this research, we established two acute pancreatitis mouse models of MAP and SAP, respectively, and through miRNA microarray analyses, we found that miR-155-5p was significantly down-regulated in these two mouse models. Afterwards, we conducted a series of in vivo and in vitro experiments to confirm that Rela (p65), a transcription factor in the NF-kB family, and tumor necrosis factor (TNF) receptor-associated factor 3 (Traf3) were targeted by miR-155-5p. In addition, we also validated that prostaglandin-endoperoxide synthase 2 (Ptgs2) acted downstream of Traf3 and was also regulated by Rela in caeruleinor TLC-S-induced AR42J cells. Thus, this study discovered a new biomarker of AP: miR-155$5 p$, which was negatively correlated with the severity of AP and regulated pancreatic acinar cell deterioration through the inhibition of the Rela/Traf3/Ptgs2 signaling pathway. 


\section{Cellular Physiology Cell Physiol Biochem 2018;51:1584-1599 \begin{tabular}{ll|l} 
and Biochemistry & $\begin{array}{l}\text { DOI: } 10.1159 / 000495648 \\
\text { Published online: } 29 \text { November } 2018\end{array}$ & $\begin{array}{l}\text { (c) The Author(s). Published by S. Karger AG, Basel } \\
\text { www.karger.com/cpb }\end{array}$ \\
\hline
\end{tabular} Liu et al.: miR-155-5p Suppresses Acute Pancreatitis Progression by Targeting Rela and Traf3}

\section{Materials and Methods}

\section{Animals}

All experiments were performed according to standard protocols approved by the Institutional Animal Care and Committee of the Hunan Normal University. The C57BL/6 mice, 6-8 weeks of age, were housed in autoclaved oblong cages at a standard ambient temperature of $20 \pm 3{ }^{\circ} \mathrm{C}$ and under a 12 -hour light-dark cycle. Animals were fed standard chow and given water ad libitum at the Animal Center of the First Affiliated Hospital of Hunan Normal University.

\section{Acute pancreatitis mouse models}

To simulate the pathology of human AP, we used caerulein and caerulein + lipopolysaccharide (LPS) to build the mild acute pancreatitis (MAP) and moderate/severe acute pancreatitis (SAP) mouse models, respectively. To construct the caerulein-induced MAP model, C57BL/6 male mice were intraperitoneally injected with $45 \mu \mathrm{g} / \mathrm{kg}$ caerulein every $6 \mathrm{~h}$ for $72 \mathrm{~h}$; to build the caerulein + lipopolysaccharide (LPS)induced SAP model, C57BL/ 6 male mice were intraperitoneally injected with $45 \mu \mathrm{g} / \mathrm{kg}$ caerulein every $6 \mathrm{~h}$ for $72 \mathrm{~h}$ and then given a single intraperitoneal injection of LPS $(10 \mathrm{mg} / \mathrm{kg})$. The control mice were injected with equal volumes of $0.9 \% \mathrm{NaCl}$ every $6 \mathrm{~h}$ for $72 \mathrm{~h}$. All induced animals were euthanized via $\mathrm{CO}_{2}$ inhalation $1 \mathrm{~h}$ later after the last caerulein, LPS or $0.9 \% \mathrm{NaCl}$ injection, and fresh blood and pancreatic acinar tissue samples were appropriately collected for analysis. Firstly we removed the blood vessels and connective tissues around the pancreas, and then cut the head and the tail of the pancreas. Afterwords, the body of the pancreas was taken out and put into the saline for washing, and then the body of the pancreas was cut open. The inner layer of the pancreas body of 3-5 mice was scraped and the total protein was extracted for western blot analysis.

\section{Patients and sample collection}

This research included 53 AP patients admitted to the Department of Hepatobiliary Surgery and 53 healthy subjects from the Center of Health Management of the Hunan Provincial People's Hospital/The First Affiliated Hospital of Hunan Normal University; the participants were recruited between 2016/10/19 and $2017 / 10 / 19$. All subjects provided signed informed consent in accordance with the declaration of Helsinki and with the permission of Ethics Committee of Hunan Normal University. The severity of the AP cases was based on the revised Atlanta Classification [18]. AP patients' peripheral blood samples were collected during the first $48 \mathrm{~h}$ after the admission from the patients' cubital vein. The serum samples from AP patients and healthy people were stored at $-80^{\circ} \mathrm{C}$ and analyzed by RT-PCR within one week after collection. In addition, during or after sample collection, the researchers recorded each of the participants' individual basic information, which was archived in detail in order to ensure that each participant's information could be clearly identified.

\section{miRNA microarray analysis}

Total RNA of peripheral serum from MAP or SAP mouse models was extracted using TRIzol reagent (Invitrogen, Carlsbad, CA, USA). miRNA microarray analysis was used to detect the differential expression of miRNAs in the peripheral blood between the MAP or SAP mice and controls.

\section{Acinar cell culture, treatment and transfection}

The rat pancreatic acinar cell line AR42J (Department of Shanghai Cell Biology, Shanghai, China) was cultured in DMEM-F/12 medium (Gibco, Grand Island, NY, USA) containing 15\% fetal bovine serum (Gibco, Grand Island, NY, USA) with $100 \mathrm{U} / \mathrm{mL}$ penicillin and $100 \mathrm{mg} / \mathrm{mL}$ streptomycin (HyClone, Logan, UT, USA) in a humidified incubator at $37^{\circ} \mathrm{C}$ with $5 \% \mathrm{CO}_{2}$ atmosphere. The AR42J cells were digested with $0.25 \%$ trypsin (HyClone) and seeded in 6-well plates $\left(1 \times 10^{6}\right.$ cells/well) after reaching $100 \%$ confluence. And then, after the cells reached $70 \%$ confluence, the cells were divided into three groups: one was treated with caerulein $(100 \mathrm{nM})$; another was treated with the natural bile acid TLC-S $(250 \mu \mathrm{M})$, and the last group was treated with L-arginine $(150 \mu \mathrm{M})$ for 45 minutes to mimic the pathological stages of AP. Subsequently, the culture medium was discarded and replaced with fresh medium, and some of them were extracted protein while the rest were transfected with $80 \mathrm{nM}$ miR-155-5p mimic (or its NC) or $100 \mathrm{nM}$ miR-155-5p inhibitor (or its NC) (Sangon Biotech, Shanghai, China) using Lipofectamine 2000 (Invitrogen, Carlsbad, CA, USA). In addition, 


\section{Cellular Physiology Cell Physiol Biochem 2018;51:1584-1599

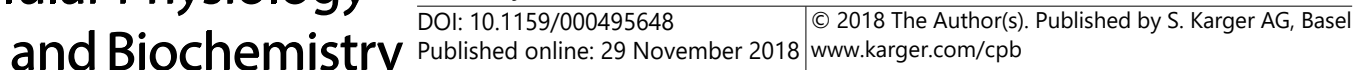 \\ Liu et al.: miR-155-5p Suppresses Acute Pancreatitis Progression by Targeting Rela and Traf3}

cells were independently transfected with $100 \mu \mathrm{M}$ helenalin or its NC (Selleck Chemicals, Houston, TX, USA) according to the manufacturer's protocol. After transfection with miR-155-5p mimic or inhibitor for 4-6 h, the transfection medium was changed with fresh normal medium, and $24 \mathrm{~h}$ later, total RNA or total proteins were extracted. In the helenalin transfections, there was no need to change the transfection medium until the extraction of total RNA or total proteins.

\section{Western blotting}

Total proteins were extracted from the pancreatic acinar tissues and the transfected AR42J cells using RIPA Lysis Buffer (Thermo Fisher Scientific, Waltham, MA, USA) supplemented with 100× proteinase inhibitor and separated by $4 \%-12 \%$ SDS-PAGE. Then, the proteins were transferred to a PVDF membrane (Merck Millipore, Billerica, MA, USA) and blocked with 5\% non-fat milk for $1.5 \mathrm{~h}$ at room temperature. The rabbit primary anti-Rela (1:1000 dilution), anti-Traf3 (1:800 dilution), anti-Ptgs2 (1:500 dilution), anti-TNF- $\alpha$ (1:750 dilution), anti-IL-6 (1:500 dilution) and anti- $\beta$-actin (1:1000 dilution) antibodies were purchased from Abcam (Cambridge, MA, USA). The secondary antibody (ZSGB-BIO, Beijing, China) was used at the dilution of 1:5000. Signals were visualized with an ECL kit (Invitrogen). The gray bands were analyzed with Image s software (NIH, Bethesda, MD, USA) to compare the expression between targeted proteins and internal controls.

\section{Quantitative real-time PCR analysis}

Total RNA was extracted from pancreatic acinar tissues or transfected AR42J cells using TRIzol reagent (Invitrogen), and $\mathrm{l} \mu \mathrm{g}$ total RNA was reverse transcribed using a reverse transcription kit (Takara, Tokyo, Japan) according to the manufacturer's protocol. Sequences of the primers were as Supplement Table 1 (For all supplemental material see www.karger.com/ 10.1159/000495648/): Reactions were performed and analyzed using the standard SYBR Green Assay protocol on a Real-Time PCR System (Roche, Basel, Switzerland). The comparative $2^{-\Delta \Delta \mathrm{Ct}}$ cycle threshold method was used for relative quantification of gene expression. Each sample was analyzed in triplicate.

\section{Dual-luciferase activity assay}

Fragments containing the 3'UTRs of mouse Rela and Traf3 were amplified from mouse genomic DNA with the following primer pairs: Rela forward, 5'-GGA GGA GCA AAC GCA GGA GCA AAA ACC ACC AGG AGA CGG AGC -3', Rela reverse 5'-CTC GAC ATG GAT CCC TGC ACA CCT TGA TCC AAA GCA ACG CTC-3'; Traf3 forward, GAG TTA GCA GCA CGC CAC AAC ACC AGA TAG CAT TAT GAT GTT-3' ${ }^{\prime}$, Traf3 reverse, $5^{\prime}$ - GTA ATG CTC AAG TGT CTA TTA GAG GAA AAT AAA GCT GAT TCA-3'. The pGL3-Rela-wild or pGL3-Traf3-wild reporters were constructed by digestion of the PCR products of 3'UTR of Rela or Traf3 with XbaI and subsequent cloning into the corresponding site of the pGL3 vector. The QuikChange II Site-Directed Mutagenesis Kit (Takara) was used to introduce mutations into the putative recognition sites of miR-155-5p, according to the manufacturer's protocol. We inserted the 3'UTR of the Rela or Traf3 DNA sequence (Rela or Traf3 3'UTR) with either an miR-155-5p mimic or an miR-155-5p inhibitor immediately downstream of the firefly or renilla luciferase gene. The normalized luciferase activity for each construct was compared to that of the pGL3 Vector control (no insert). Then, $500 \mathrm{ng}$ of the Luciferase reporter, $80 \mathrm{nM}$ miRNA-155-5p mimic or its NC, $100 \mathrm{nM}$ miRNA-155-5p inhibitor or its NC and $30 \mathrm{ng}$ of the pRL-TK Renilla Luciferase Reporter Vector (Promega, Madison, WI, USA) were added to each 24 -well plate $\left(4 \sim 5 \times 10^{4}\right.$ cells/well) for transfection. After $48 \mathrm{~h}$, the cells were collected and the luciferase activity was measured with the Dual-Luciferase ${ }^{\circ}$ Reporter Assay system (Promega).

\section{Statistical Analysis}

Data are presented as the mean \pm SD. All statistical analyses were performed using the SPSS17.0 software. Differences between groups were compared using Student's t-test for two groups or one-way ANOVA to compare three or more groups. Categorical variables were compared with the Fisher 2-tailed exact test. Statistical significance was accepted at $P<0.05$. 
Liu et al.: miR-155-5p Suppresses Acute Pancreatitis Progression by Targeting Rela and

Traf3

\section{Results}

\section{MiRNA microarray analysis showed the differential expressions of miRNAs}

To investigate the different stages of $\mathrm{AP}$, we built mouse models of MAP and SAP and then isolated serum from the peripheral blood of the mice. We used an miRNA microarray to analyze the differential expression of miRNAs in experimental and control mice. In comparing MAP and control mice, we found 64 miRNAs from the peripheral blood that showed obvious, significant differences. Among the 64 miRNAs, 34 miRNAs were up-regulated and 30 miRNAs were down-regulated compared to the control. Notably, miR-455-5p was the most up-regulated (elevated approximately 21-fold) while the expression of miR155-5p was the most downregulated (declined nearly 17-fold) (Table 1). When we compared the relative miRNA expression between SAP and control mice, we clearly found that the expression of miR-302-3p was the most up-regulated (elevated approximately 22-fold), while miR-155-5p was still the most downregulated miRNA (declined 20 -fold) (Table 2).
Table 1. MiRNA microarray analysis of differentially expressed microRNAs in the caerulein-induced pancreatitis model and control mice (pancreatitis mice/control mice)

\begin{tabular}{|c|c|c|c|c|c|}
\hline MicroRNAs & Up-regulated & $\begin{array}{c}\mathrm{P} \\
\text { (value) }\end{array}$ & MicroRNAs & Down-regulated & $\begin{array}{c}P \\
\text { (value) }\end{array}$ \\
\hline mmu-miR-455-5p & 21.38 & 0.0017 & mmu-miR-195 & 0.91 & 0.0013 \\
\hline mmu-miR-302-3p & 19.74 & 0.0078 & mmu-miR-445-5p & 0.87 & 0.0301 \\
\hline mmu-miR-373-3p & 17.65 & 0.0197 & mmu-miR-1306 & 0.77 & 0.0093 \\
\hline mmu-miR-122 & 15.77 & 0.0213 & mmu-miR-130a-3p & 0.72 & 0.0193 \\
\hline mmu-miR-808 & 15.33 & 0.0108 & mmu-miR-301a & 0.66 & 0.0219 \\
\hline mmu-miR-148 & 14.88 & 0.0079 & mmu-miR-301b & 0.61 & 0.0091 \\
\hline mmu-miR-138-5p & 14.23 & 0.0041 & mmu-miR-351-5p & 0.55 & 0.0312 \\
\hline mmu-miR-29-3p & 13.77 & 0.0037 & mmu-miR-125b-5p & 0.52 & 0.0401 \\
\hline mmu-miR-223 & 12.98 & 0.0073 & mmu-miR-125a-5p & 0.47 & 0.0047 \\
\hline mmu-miR-182 & 12.57 & 0.0409 & mmu-miR-99 & 0.43 & 0.0012 \\
\hline mmu-miR-194-5p & 11.38 & 0.0078 & mmu-miR-100 & 0.39 & 0.0079 \\
\hline mmu-miR-506-3p & 11.75 & 0.0018 & mmu-miR-208-3p & 0.38 & 0.0016 \\
\hline$m m u-m i R-372-3 p$ & 9.11 & 0.0051 & mmu-miR-499-5p & 0.33 & 0.0073 \\
\hline mmu-miR-520a-3p & 8.11 & 0.0182 & mmu-miR-27-3p & 0.31 & 0.0147 \\
\hline mmu-miR-520d-3p & 7.31 & 0.0047 & mmu-miR-33-5p & 0.28 & 0.0045 \\
\hline mmu-miR-520e & 7.01 & 0.0301 & mmu-miR-295 & 0.25 & 0.0078 \\
\hline mmu-miR-1306-5p & 5.89 & 0.0072 & mmu-miR-23 & 0.23 & 0.0128 \\
\hline mmu-miR-7a-5p & 5.12 & 0.0094 & mmu-miR-100 & 0.21 & 0.0327 \\
\hline mmu-miR-490 & 4.91 & 0.0287 & mmu-miR-19-3p & 0.20 & 0.0421 \\
\hline mmu-miR-802 & 4.18 & 0.0092 & mmu-miR-338 & 0.19 & 0.0219 \\
\hline mmu-miR-194 & 3.98 & 0.0731 & mmu-miR-181 & 0.18 & 0.0128 \\
\hline mmu-miR-140 & 3.54 & 0.0083 & mmu-miR-130 & 0.17 & 0.0271 \\
\hline mmu-miR-125-5P & 3.33 & 0.0072 & mmu-miR-301-3p & 0.17 & 0.0231 \\
\hline mmu-miR-132 & 3.11 & 0.0064 & mmu-miR-204-5p & 0.16 & 0.0317 \\
\hline mmu-miR-194 & 2.98 & 0.0048 & $m m u-m i R-211-5 p$ & 0.15 & 0.0187 \\
\hline mmu-miR-205-5p & 2.56 & 0.0317 & mmu-miR-351 & 0.13 & 0.0035 \\
\hline mmu-miR-212 & 2.22 & 0.0019 & mmu-miR-125 & 0.11 & 0.0013 \\
\hline mmu-miR-122 & 2.14 & 0.0052 & mmu-miR-363 & 0.10 & 0.0062 \\
\hline mmu-miR-15 & 2.13 & 0.0081 & mmu-miR-367 & 0.09 & 0.0023 \\
\hline mmu-miR-16 & 2.03 & 0.0017 & mmu-miR-155-5p & 0.06 & 0.0011 \\
\hline mmu-miR-302 & 1.99 & 0.0057 & & & \\
\hline mmu-miR-138 & 1.78 & 0.0073 & & & \\
\hline mmu-miR-22 & 1.34 & 0.0049 & & & \\
\hline mmu-miR-13-3p & 1.12 & 0.0209 & & & \\
\hline
\end{tabular}

Table 2. MiRNA microarray analysis of differentially expressed microRNAs in TCL-S-induced pancreatitis model and control mice (pancreatitis mice/control mice)

\begin{tabular}{|c|c|c|c|c|c|}
\hline MicroRNAs & Up-regulated & $\begin{array}{c}\mathrm{P} \\
\text { (value) }\end{array}$ & MicroRNAs & Down-regulated & $\begin{array}{c}P \\
\text { (value) }\end{array}$ \\
\hline mmu-miR-302-3p & 22.33 & 0.0032 & mmu-miR-445-5p & 0.91 & 0.0109 \\
\hline$m m u-m i R-455-5 p$ & 21.78 & 0.0149 & mmu-miR-195 & 0.88 & 0.0216 \\
\hline mmu-miR-373-3p & 20.44 & 0.0021 & mmu-miR-1306 & 0.85 & 0.0093 \\
\hline mmu-miR-122 & 19.89 & 0.0078 & mmu-miR-130a-3p & 0.72 & 0.0071 \\
\hline mmu-miR-808 & 17.23 & 0.0172 & mmu-miR-301a & 0.71 & 0.0341 \\
\hline mmu-miR-138-5p & 14.67 & 0.0209 & mmu-miR-301b & 0.66 & 0.0182 \\
\hline mmu-miR-29-3p & 14.12 & 0.0182 & mmu-miR-125b-5p & 0.58 & 0.0117 \\
\hline mmu-miR-148 & 13.91 & 0.0197 & mmu-miR-351-5p & 0.52 & 0.0028 \\
\hline mmu-miR-223 & 13.27 & 0.0213 & mmu-miR-125a-5p & 0.45 & 0.0419 \\
\hline mmu-miR-182 & 12.92 & 0.0172 & mmu-miR-99 & 0.41 & 0.0192 \\
\hline mmu-miR-506-3p & 12.56 & 0.0091 & mmu-miR-100 & 0.36 & 0.0075 \\
\hline mmu-miR-372-3p & 11.78 & 0.0413 & mmu-miR-208-3p & 0.34 & 0.0112 \\
\hline mmu-miR-194-5p & 10.78 & 0.0117 & mmu-miR-27-3p & 0.33 & 0.0213 \\
\hline mmu-miR-520a-3p & 10.67 & 0.0075 & mmu-miR-499-5p & 0.31 & 0.0173 \\
\hline mmu-miR-520d-3p & 9.33 & 0.0097 & mmu-miR-33-5p & 0.27 & 0.0053 \\
\hline mmu-miR-520e & 8.23 & 0.0182 & mmu-miR-100 & 0.26 & 0.0319 \\
\hline mmu-miR-1306-5p & 7.67 & 0.0041 & mmu-miR-19-3p & 0.24 & 0.0401 \\
\hline mmu-miR-7a-5p & 7.01 & 0.0314 & mmu-miR-338 & 0.23 & 0.0047 \\
\hline mmu-miR-490 & 6.54 & 0.0019 & mmu-miR-295 & 0.22 & 0.0053 \\
\hline mmu-miR-802 & 5.78 & 0.0064 & mmu-miR-23 & 0.19 & 0.0072 \\
\hline mmu-miR-194 & 5.01 & 0.0182 & mmu-miR-211-5p & 0.17 & 0.0126 \\
\hline mmu-miR-140 & 4.78 & 0.0205 & mmu-miR-181 & 0.16 & 0.0277 \\
\hline mmu-miR-122 & 4.77 & 0.0311 & mmu-miR-130 & 0.14 & 0.0175 \\
\hline mmu-miR-125-5P & 4.09 & 0.0102 & mmu-miR-301-3p & 0.14 & 0.0091 \\
\hline mmu-miR-132 & 3.95 & 0.0081 & mmu-miR-204-5p & 0.13 & 0.0192 \\
\hline mmu-miR-15 & 3.77 & 0.0226 & mmu-miR-351 & 0.12 & 0.0213 \\
\hline mmu-miR-194 & 3.11 & 0.0072 & mmu-miR-125 & 0.09 & 0.0061 \\
\hline$m m u-m i R-205-5 p$ & 2.98 & 0.0271 & mmu-miR-367 & 0.08 & 0.0143 \\
\hline mmu-miR-16 & 2.87 & 0.0317 & mmu-miR-363 & 0.07 & 0.0093 \\
\hline mmu-miR-212 & 2.11 & 0.0219 & mmu-miR-155-5p & 0.05 & 0.0049 \\
\hline mmu-miR-302 & 1.88 & 0.0108 & & & \\
\hline mmu-miR-138 & 1.78 & 0.0411 & & & \\
\hline mmu-miR-22 & 1.56 & 0.0079 & & & \\
\hline mmu-miR-13-3p & 1.12 & 0.0127 & & & \\
\hline
\end{tabular}


Liu et al.: miR-155-5p Suppresses Acute Pancreatitis Progression by Targeting Rela and Traf3

Fig. 1. Combined analysis with the TargetScan software and dual-luciferase gene reporter assays to confirm that Rela was targeted by miR-1555p. A. The predicted c o r r e s p o n d in g sequences of miR-155$5 \mathrm{p}$ and 3'UTR of Rela mRNA. B. The sequences on the top refer to the wild-type matching sequences of miR-155$5 p$ and 3'UTR of Rela mRNA, while the ones at the bottom refer to the mutated matching sequences of miR155-5p and 3'UTR of Rela mRNA. C. When miR-155-5p mimic and Rela 3'UTR wild type (WT) were cotransfected into AR42J cells, the luciferase activity obviously decreased; however, the luciferase activity did not significantly change when miR-155-5p mimic and Rela 3'UTR mutant were co-transfected into AR42J cells. D. The luciferase activity was significantly promoted when miR-155-5p inhibitor and Rela 3'UTR were co-transfected

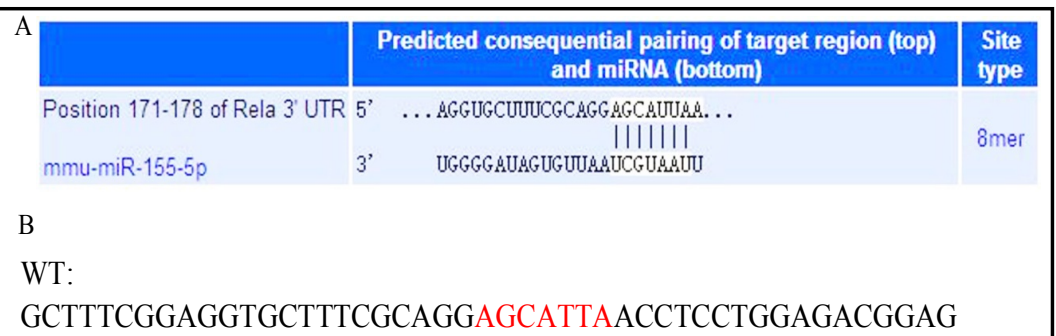

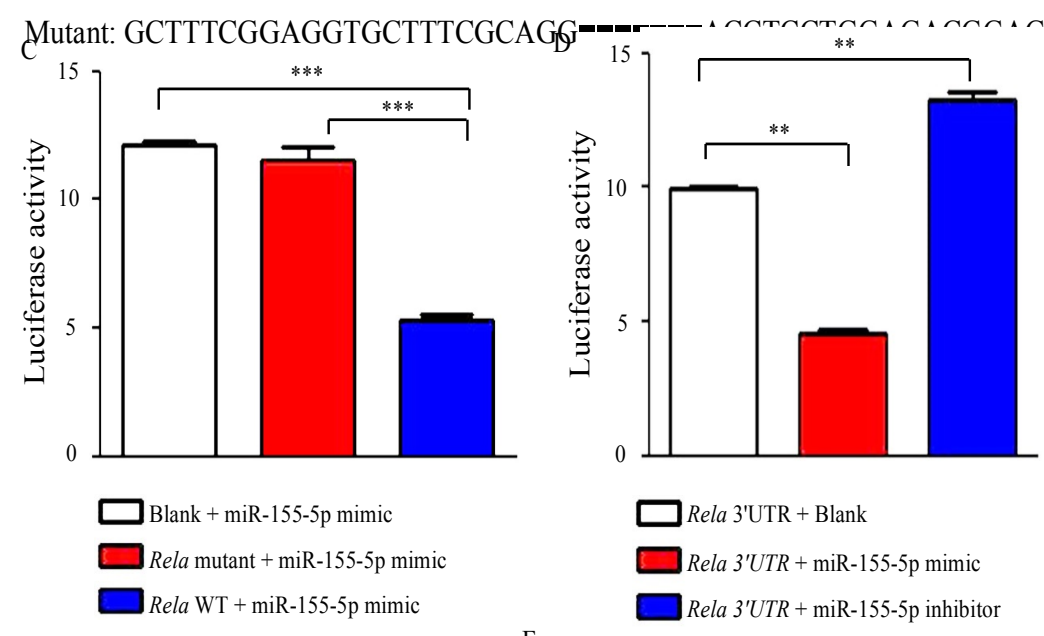
E $\mathrm{F}$

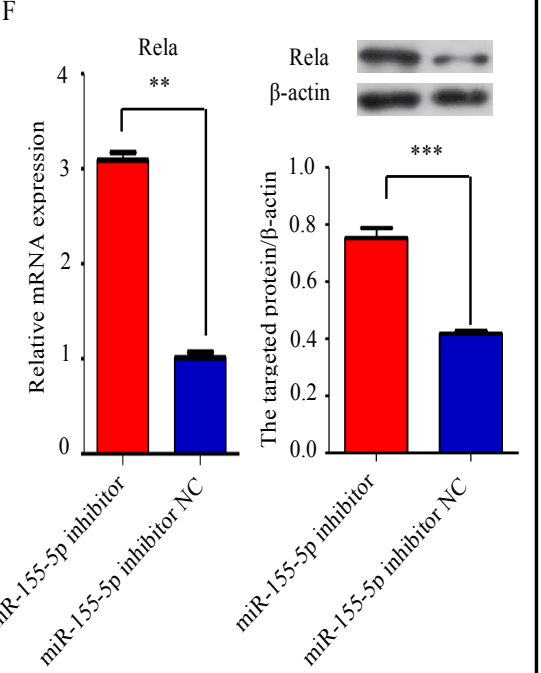
into AR42J cells, but the results were the opposite when miR-155-5p mimic and Rela 3'UTR were co-transfected into AR42J cells. E, F. After transfection of AR42J cells with miR-155-5p mimic, both at the mRNA and protein level, the expression of Rela was reduced compared with the $\mathrm{NC}$, and the results were reversed upon transfection with miR-1555 p inhibitor. ${ }^{*} \mathrm{P}<0.05,{ }^{* *} \mathrm{P}<0.01,{ }^{* * *} \mathrm{P}<0.001$.

Combined analysis with the TargetScan software, dual-luciferase gene reporter assays and transfection of AR42J cells to confirm that Rela was targeted by miR-155-5p

Since the expression of miR-155-5p was the lowest in both MAP and SAP mice, compared to controls, this miRNA was selected for further analysis in this research. Thus, in order to determine the potential target genes of miR-155-5p that could be related to pancreatitis, we first confirmed that Rela was one of the genes targeted by miR-155-5p and then analyzed the alignment of their sequences using the TargetScan software (Fig. 1A and 1B). In addition, we transfected the AR42J cells with the corresponding sequences of Rela wild-type (WT) mRNA 
and miR-155-5p and found that the luciferase activity significantly decreased. However, the luciferase activity did not change significantly when Rela mutant mRNA and miR-155$5 p$ were transfected into the AR42J cells (Fig. 1C). Importantly, the luciferase activity was relatively up-regulated upon transfection of the corresponding sequences of miR-155-5p inhibitor and the 3'UTR of Rela mRNA into the AR42J cells. Furthermore, we obtained the opposite results after transfection of miR-155-5p mimic (Fig. 1D). In L-arginine-induced AR42J cells transfected with miR-155-5p mimic, the expression of Rela decreased at both the transcriptional and post-transcriptional level (Fig. 1E). However, the results were the opposite after transfection with miR-155-5p inhibitor (Fig. 1F).

Fig. 2. Through TargetScan software and dual-luciferase genes reporter assays to verify that Traf3 was also targeted by miR-155-5p. A. The predicted corresponding sequences of miR155-5p and 3'UTR of Traf3 mRNA. B. The sequences on the top refer to the wild-type matching sequences of miR-155-5p and 3'UTR of Traf3 mRNA, while the ones at the bottom refer to the mutated matching sequences of miR-155-5p and 3'UTR of Traf3 mRNA. C. The luciferase activity was decreased when miR155-5p mimic and Traf3 3'UTR wild type (WT) were co-transfected into AR42J cells, however, the luciferase activity was no change after miR155-5p mimic and Traf3 3'UTR mutant were cotransfected into AR42J cells. D. The luciferase activity was significantly promoted when miR$155-5 \mathrm{p}$ inhibitor and

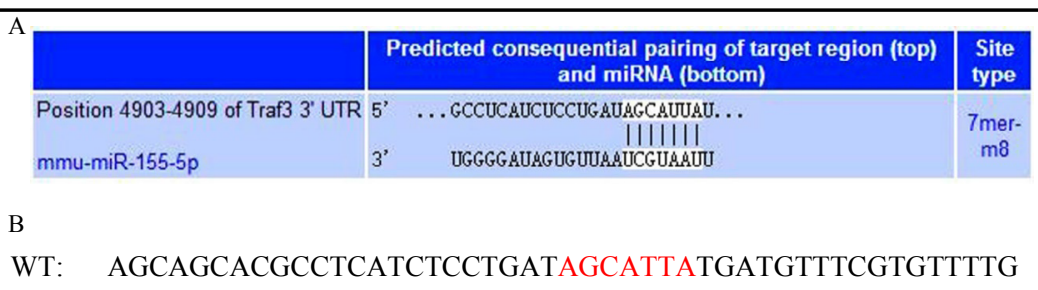

Mutant: AGCAGCACGCCTCATCTCCTGAT-------TGATGTTTCGTGTTTTG

$\mathrm{C}$
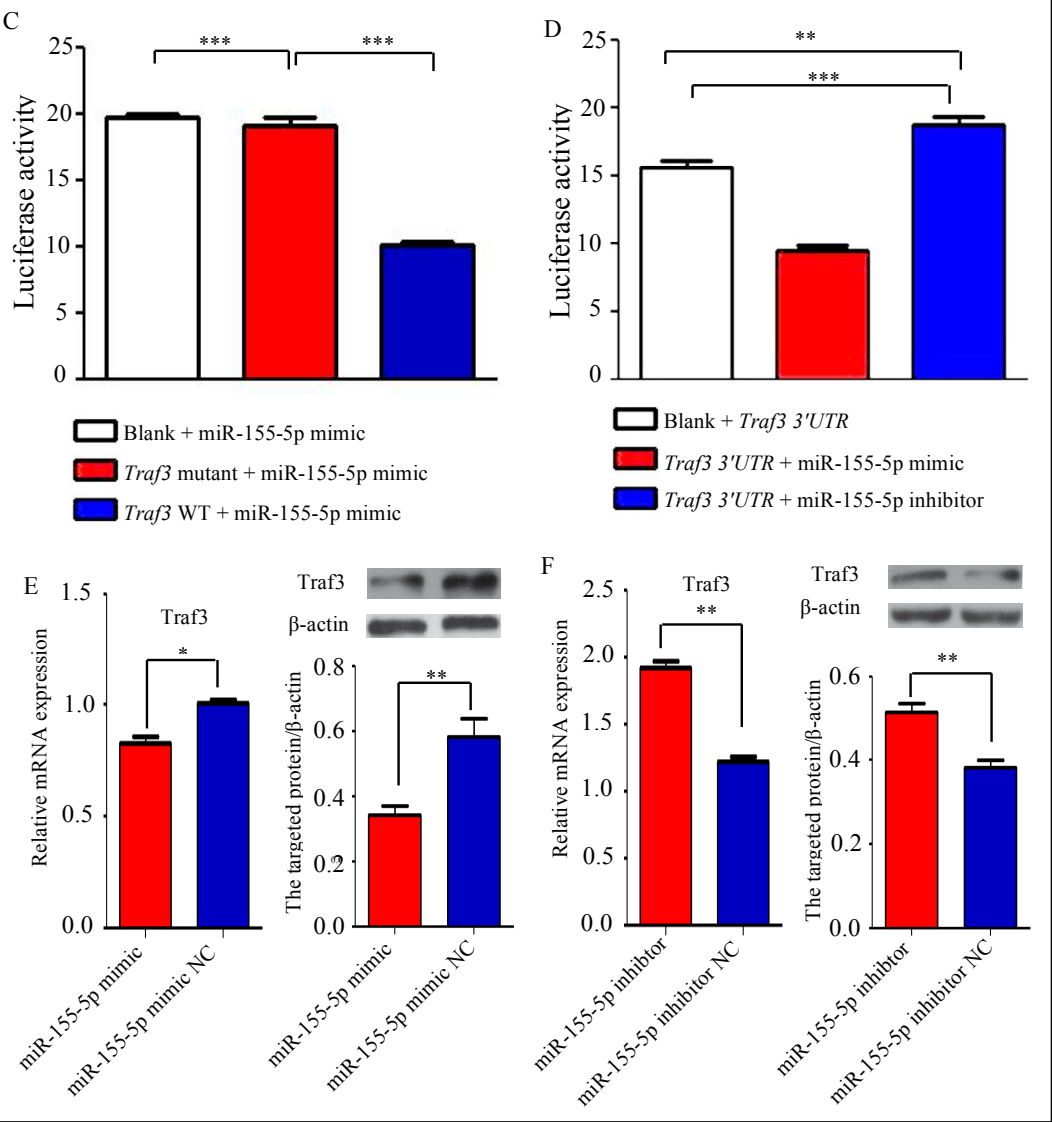

Traf 3'UTR were co-

transfected into AR42J cells, but the results were the opposite when miR-155-5p mimic and Traf3 3'UTR were co-transfected into AR42J cells. E, F. After transfection of AR42J cells with miR-155-5p mimic, the expression of Traf3 was reduced at both the mRNA and protein level compared with the NC; however, the results were reversed upon transfection with miR-155-5p inhibitor. ${ }^{*} \mathrm{P}<0.05,{ }^{* *} \mathrm{P}<0.01,{ }^{* * *} \mathrm{P}<0.001$. 


\section{Cellular Physiology Cell Physiol Biochem 2018;51:1584-1599 \begin{tabular}{l|l|l|l|l}
\hline DOI: 10.1159/000495648 & 2018 The Author(s). Published by S. Karger AG, Basel
\end{tabular} Published onlıne: 29 November 2018 www.karger.com/cpb \\ Liu et al.: miR-155-5p Suppresses Acute Pancreatitis Progression by Targeting Rela and Traf3}

Through TargetScan software, and dual-luciferase gene reporter assays and transfection of AR42J cells to verify that Traf3 was also targeted by miR-155-5p

Through targetscan software, we also found the binding sequences of miR-155-5p and 3'UTR of Traf3 (Fig. 2A and 2B). Afterwards, the corresponding sequences of Traf3 WT mRNA and miR-155-5p were transfected into the cells, and found the luciferase activity was decreased. However, after Traf3 mutant mRNA and miR-155-5p were co-transfected into the cells, the luciferase activity was no statistical difference (Fig. 2C). In addition, the luciferase activity was elevated as the binding sequences of miR-155-5p inhibitor and the 3'UTR of Traf3 were transfected into the cells, but the results were opposite after miR-155-5p mimic replaced miR-155-5p inhibitor (Fig. 2D). In AR42J cells induced with L-arginine, which was transfected with miR-155-5p mimic, the expression of Traf3 was declined at both mRNA and protein level (Fig. 2E). However, the results turned to be reverse after miR-155-5p inhibitor was transfected into the cells (Fig. 2F).

The expression of TNF- $\alpha$ and IL-6 and Rela/Traf3/Ptgs2 were elevated after AR42J cells were induced by caerulein- or L-arginine or TLC-S

It has been found that the expression levels of TNF- $\alpha$ and IL- 6 were positively correlated to the severity of AP $[18,19]$. After the AR42J cells were induced by caerulein- or L-arginine or TLC-S for 45 minutes, the total protein and RNA were extracted, and through WB analysis, we found that the expression of Rela, Traf3 and Ptgs 2 were highest in TLC-S induced cells, followed by in caerulein group and the control group (Fig. 3A and 3B). Through RT-PCR analysis, the relative expression of $T N F-\alpha$ and $I L-6$ were significantly elevated in caerulein- or L-arginine or TLC-S group compared with in control group. Furthermore, the expression of TNF- $\alpha$ and $I L-6$ were higher in TLC-S group than in caerulein and L-arginine group between which there was no statistical differences (Fig. 3C).

Fig. 3. The expression of TNF- $\alpha$, IL-6 and Rela/ Traf3/Ptgs2 were upregulated in AR42J cells induced by caerulein- or L-arginine or TLC-S. A, B. After the AR42J cells were induced by caerulein or TLC-S respectively, the expression of Rela/Traf3/ Ptgs2 were significantly promoted in comparison with the control group. In addition, the expression of Rela/Traf3/Ptgs2 were higher in TLC-S group than in caerulein group. C. The expression of TNF- $\alpha$ and IL-6 were statistically higher in TLC-S group than in caerulein and L-arginine group between which there was no statistical significance. ${ }^{*} \mathrm{P}<0.05,{ }^{* *} \mathrm{P}$ $<0.01,{ }^{* * *} \mathrm{P}<0.001$.

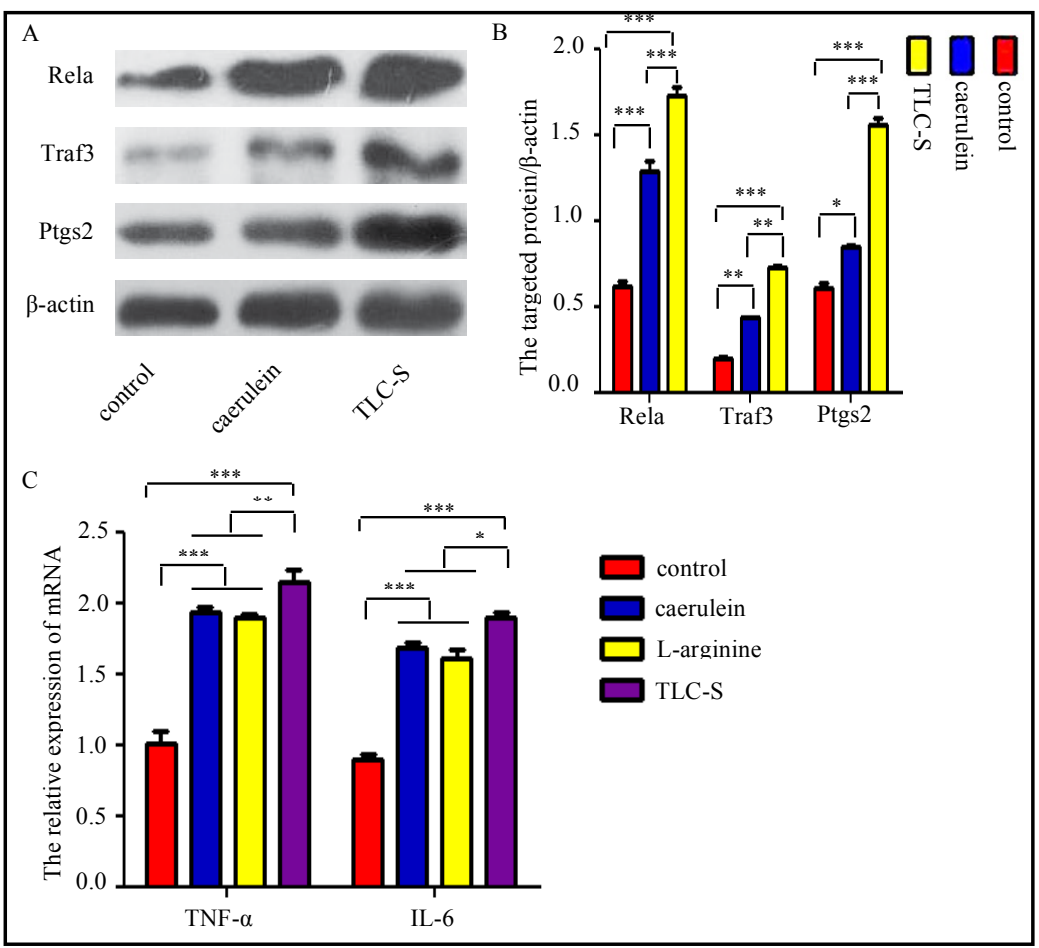


Liu et al.: miR-155-5p Suppresses Acute Pancreatitis Progression by Targeting Rela and Traf3

The expression of Rela, Traf3 and Ptgs2 in pancreatic tissues from caerulein- or caerulein + LPS-induced mice

We extracted total protein from pancreas tissues in control, caerulein- and caerulein + LPS-induced mice, and through western blotting (WB) analysis, it was found that the expression of inflammatory factors of TNF- $\alpha$ and IL- 6 was significantly promoted in caerulein induced MAP and caerulein + LPS-induced SAP mice compared to in control mice. Furthermore, the expression of TNF- $\alpha$ and IL- 6 was higher in SAP mice than in MAP mice (Fig. 4A and 4B). We found that the expression of Rela, Traf3 and Ptgs2 was obviously higher in caerulein- and caerulein + LPS-induced MAP and SAP mice respectively compared to control mice (Fig. 4C and 4D). However, at the mRNA level, the expression of Rela, Traf3 and Ptgs2 did not show any significant difference (Fig. 4E). In addition, we also extracted the total RNA from the mice pancreas tissues and, after reverse transcription and through RT-PCR, we found that the relative expression of miR-155-5p was significantly lower in caerulein and caerulein + LPS-induced MAP and SAP mice respectively compared to control mice (Fig. 4F).

Fig. 4. The expression of Rela, Traf3 and Ptgs2 in pancreatic tissues of the MAP and SAP mouse models. A, B. In caerulein induced MAP and caerulein+LPS induced SAP mice models, the expression of inflammatory factors of TNF- $\alpha$ and IL-6 in pancreatic tissues were highest in SAP mice, followed by in MAP and control mice. C, D. The expression of Rela and Traf3 was significantly promoted in the pancreatic tissues from MAP and SAP mice in comparison with in the control mice. In addition, Rela and Traf3 expression was higher in the pancreatic tissues of SAP mice than in MAP mice. E. No statistical differences were found in comparing with the mRNA expression of Rela, Traf3 and Ptgs 2 in the pancreatic tissues of control, MAP and SAP mice. F. When comparing the relative expression of miR155-5p in control, MAP and SAP mice, miR-155-5p was expressed the highest in control mice and the lowest in SAP mice.
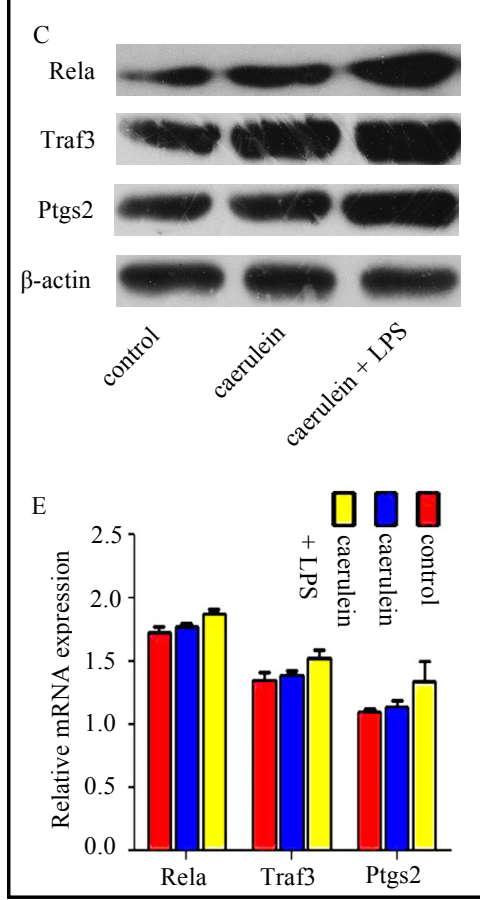
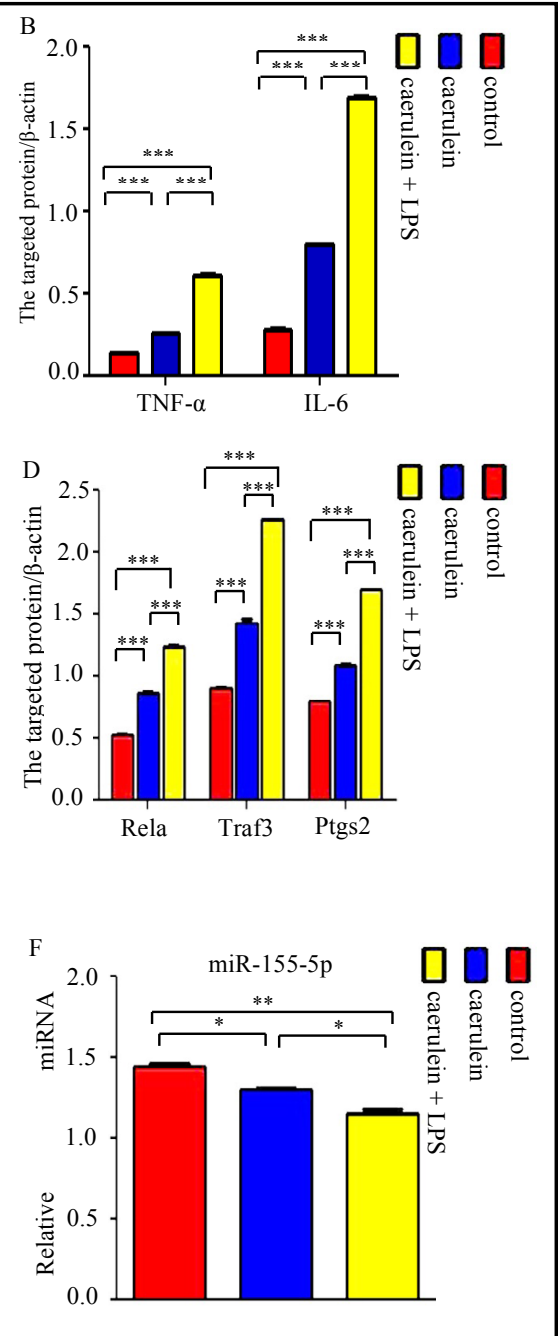
Liu et al.: miR-155-5p Suppresses Acute Pancreatitis Progression by Targeting Rela and

Traf3

The expression of Rela, Traf3 and Ptgs2 in AR42J cells after treatment with caerulein or TLC-S and transfection with miR-155-5p mimic or inhibitor

We simulated MAP or SAP in AR42J cells via incubation with caerulein or TLC-S, respectively, and then we transfected the cells with miR-155-5p mimic or inhibitor. In miR155-5p mimic group, we found that the relative expression of Rela, Traf3 and Ptgs 2 protein notably declined compared with the NC group, in both the caerulein- and TLC-S-induced AR42J cells (Fig. 5A and 5C). However, in miR-155-5p inhibitor group, the relative expression of Rela, Traf3 and Ptgs 2 protein was significantly promoted in comparison with the NC group, in both the caerulein- and TLC-S-induced AR42J cells (Fig. 5B and 5D). Notably, at the transcriptional level, the relative expression of Rela and Traf3 was significantly downregulated in miR-155-5p mimic group, in both the caerulein- and TLC-S-induced AR42J cells (Fig. 5E), while the results were the opposite in miR-155-5p inhibitor group (Fig. 5F).

Fig. 5. The expression of Rela, Traf3 and Ptgs2 in caerulein- or TLC-Sinduced AP. A, C. In both the caerulein and TLC-S group, the expression of Rela, Traf3 and Ptgs2 decreased in miR-155$5 \mathrm{p}$ mimic-transfected AR42J cells compared with the NC. B, D. In both the caerulein and TLC-S group, the expression of Rela, Traf3 and Ptgs2 increased in miR-155$5 \mathrm{p}$ inhibitor-transfected AR42J cells, compared with the NC. E. After transfection of the AR42J cells with miR-155-5p mimic, the expression of Rela and Traf3 mRNAs was also reduced in both the caerulein and TLC-S group compared with the NC. F. After transfection of AR42J cells with miR-155-5p inhibitor, the expression of Rela and Traf3 mRNAs also increased in both the caerulein and TLC-S group compared with the NC. ${ }^{*} \mathrm{P}$ $<0.05,{ }^{* *} \mathrm{P}<0.01,{ }^{* * *} \mathrm{P}<$ 0.001 .

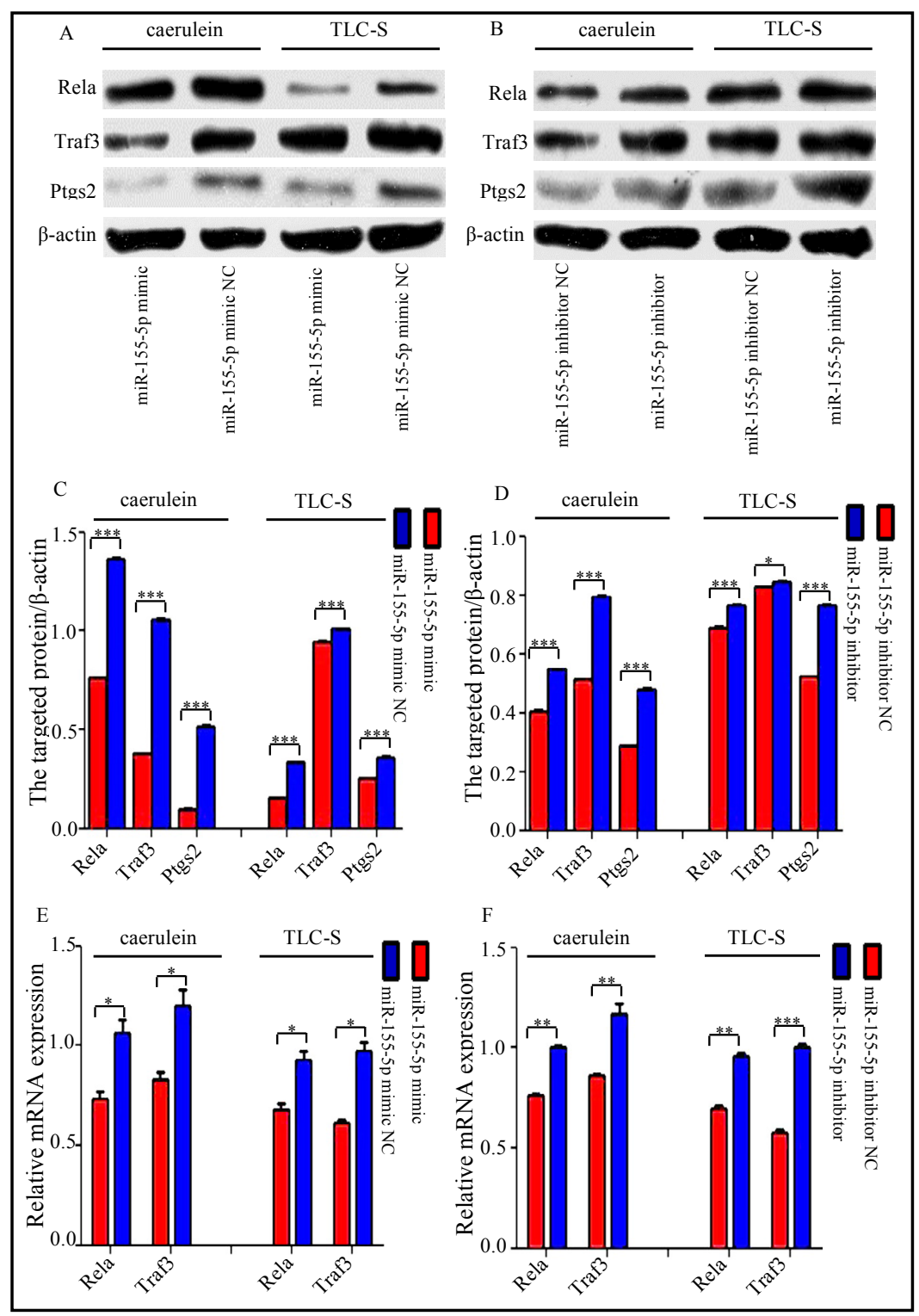


Fig. 6. The relative expression of Rela and downstream proteins after transfection of AR42J cells with helenalin in caerulein- or caerulein + LPS-induced MAP or SAP. A. After transfection of AR42J cells with helenalin, the expression of Rela mRNA was significantly reduced in both the caerulein and caerulein + LPS group. B. After transfection of AR42J cells with helenalin, the expression of Traf3 and Ptgs2 mRNA did not significantly change in either the caerulein or caerulein + LPS group. C, D. After transfection of AR42J cells with helenalin, at the protein level, the expression of Rela, Traf3 and Ptgs2 decreased in comparison with the $\mathrm{NC}$ in both the caerulein and caerulein + LPS group. E. After transfection of AR42J cells with helenalin, the expression of miR-155-5p did not significantly change compared with the NC. ${ }^{*} \mathrm{P} 0.05,{ }^{* *} \mathrm{P}<0.01,{ }^{* * *} \mathrm{P}<$ 0.001 .
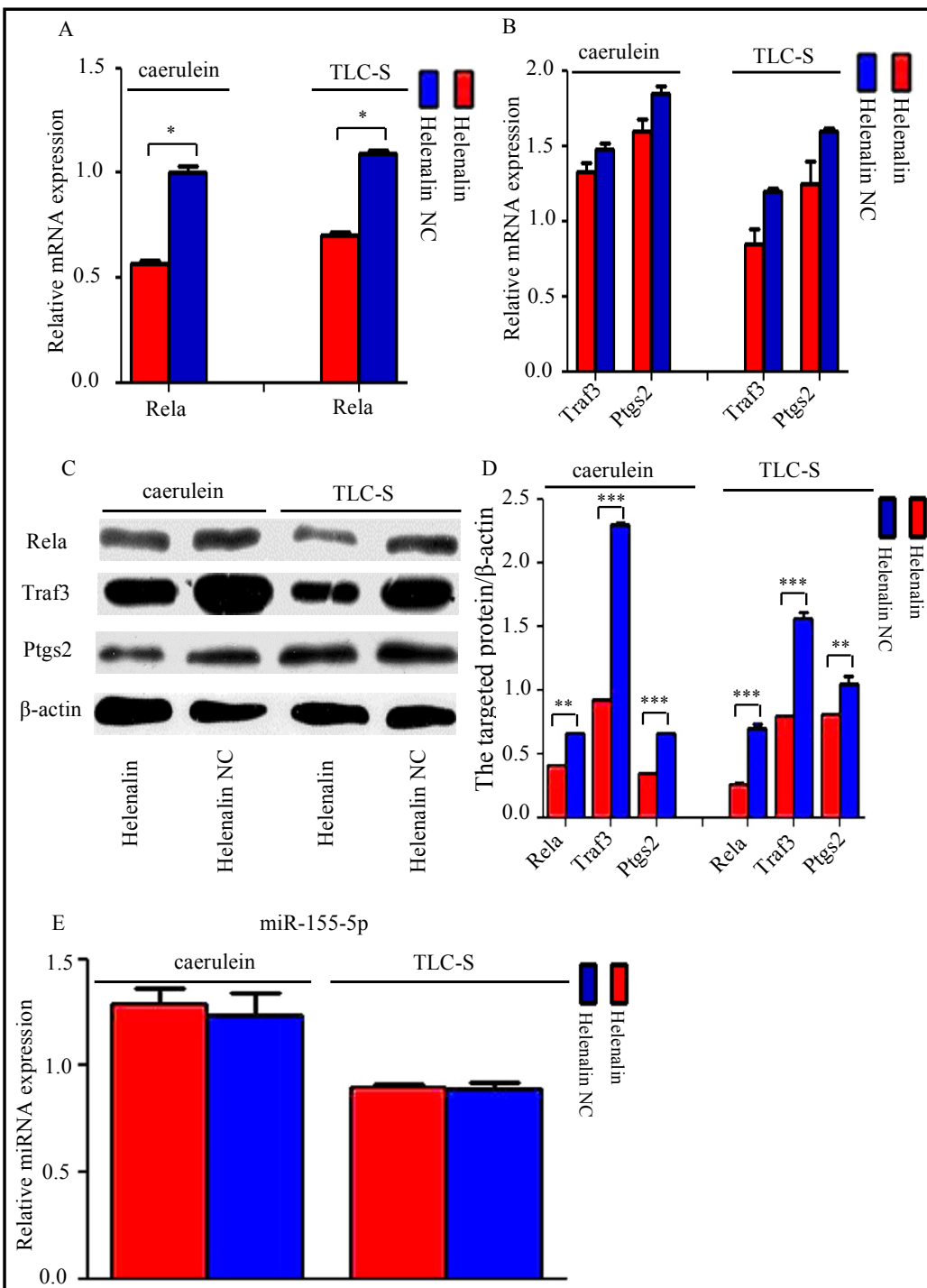

The expression of Rela, Traf3 and Ptgs 2 and miR-155-5p in AR42J cells after treatment with caerulein or TLC-S and transfection with helenalin

We transfected the Rela inhibitor helenalin into AR42J cells treated with caerulein or TLC-S and found that the expression of Rela was significantly downregulated at the mRNA level (Fig. 6A). However, the expression of Traf3 and Ptgs2 Table 3. The basic characteristics of the research subjects. Values are given as the mean \pm standard deviation (SD); ${ }^{a}$ Fisher 2-tailed exact test; b Student's t test

\begin{tabular}{lccc}
\hline Parameters & MAP $(\mathrm{n}=29)$ & SAP $(\mathrm{n}=24)$ & $\mathrm{P}($ value $)$ \\
\hline Age (years) & $52 \pm 14$ & $52 \pm 15$ & $0.4337 \mathrm{~b}$ \\
Male & $15(51.7 \%)$ & $13(54.2 \%)$ & $0.4011^{\mathrm{a}}$ \\
Female & $14(48.3 \%)$ & $11(45.8 \%)$ & \\
Gallbladder lithiasis & $13(44.8 \%)$ & $10(41.7 \%)$ & $0.3122^{\mathrm{a}}$ \\
Hypertriglyceridemia & $4(13.8 \%)$ & $3(12.5 \%)$ & 0.3097 a \\
Alcoholism & $11(37.9 \%)$ & $9(37.5 \%)$ & 0.2978 a \\
Other & $1(3.5 \%)$ & $2(8.3 \%)$ & $0.5022^{\mathrm{a}}$ \\
\hline
\end{tabular}
did not significantly change (Fig. 6B). At the protein level, the relative expression of Rela, Traf3 and Ptgs 2 obviously decreased in the helenalin group in comparison with the NC group, in both the caerulein- and TLC-S-induced AR42J cells (Fig. 6C and 6D). In addition, we also detected the relative expression of miR-155-5p in AR42J cells, and miR-155-5p expression was no change in both the caerulein- and TLC-S-induced cells transfected with helenalin and in the NC group (Fig. 6E). 


\section{Cellular Physiology \begin{tabular}{l|l|l} 
and Biochemistry Published online: 29 November 2018 & $\begin{array}{l}\text { ( 2018 The Author(s). Published by S. Karger AG, Basel } \\
\text { www.karger.com/cpb }\end{array}$ \\
\hline
\end{tabular} \\ Liu et al.: miR-155-5p Suppresses Acute Pancreatitis Progression by Targeting Rela and Traf3}

The relative expression of $m i R-155-5 p$ in AP patients and healthy subjects

To detect the relative expression of miR-155-5p in AP patients and healthy subjects, we collected serum from $53 \mathrm{AP}$ patients and 53 healthy subjects. Among the 53 AP patients, 29 were diagnosed with MAP and 24 with SAP, according to the revised Atlanta Classification [20]. In regard to age, gender, and risk factors related to pancreatitis, we did not find any significant difference between MAP and SAP (Table 3). Through qRT-PCR analysis, we found that the relative expression of miR-155-5p was notably lower in AP patients in comparison with healthy subjects (Fig. 7A). Importantly, the decrease in the relative expression of miR-155-5p was more obvious in SAP patients compared with MAP patients (Fig. 7B).

\section{Discussion}

In previous studies, researchers have focused their attention on the dysregulation of gene transcription and translation in the process of pancreatitis. However, few studies have demonstrated the important relationship between miRNAs and AP. Nonetheless, miRNAs exert vital roles in the occurrence and development of AP. A great deal of evidence has illustrated that miRNAs' primary function is to suppress the expression of downstream target genes by interacting with the 3'UTR of their mRNA[21-23], and therefore, miRNAs play

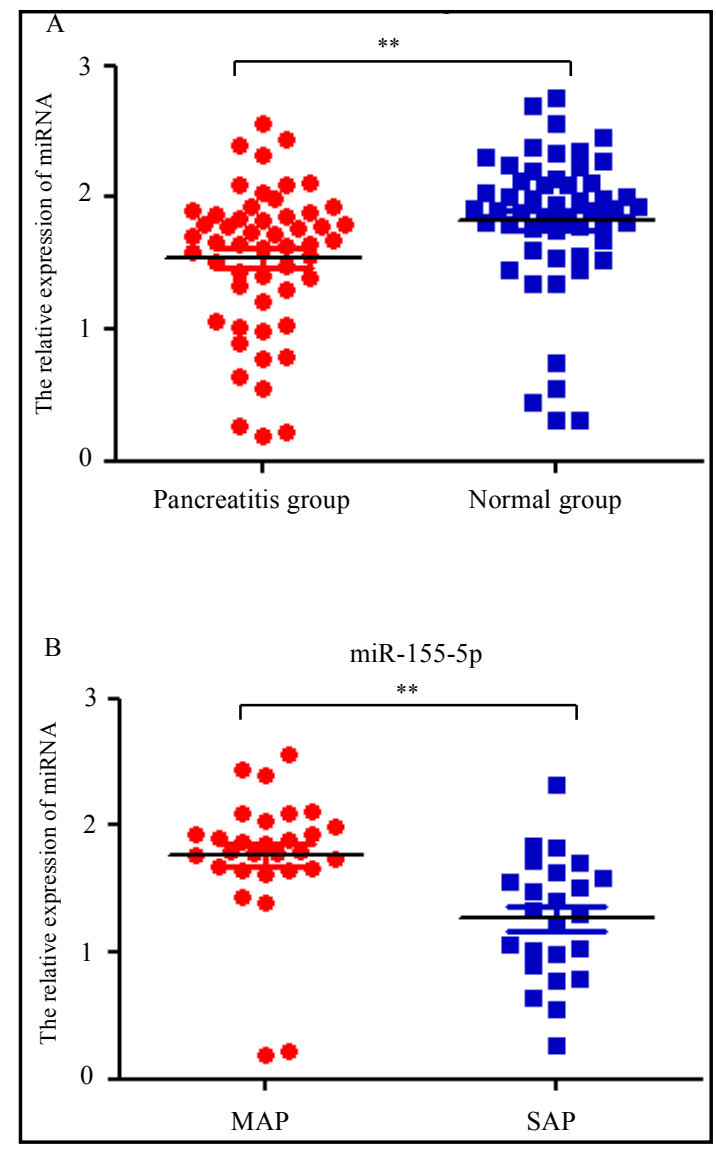

Fig. 7. The relative expression of miR-155-5p in the normal and pancreatitis groups. A. Compared with the healthy patients group $(n=53)$, the relative expression of miR-155-5p was significantly decreased in the pancreatitis group $(n=53)$. B. In comparison with the MAP patients group $(n=29)$, the relative expression of miR-155-5p was significantly reduced in the SAP patients group $(\mathrm{n}=24) .{ }^{*} \mathrm{P} 0.05$, $* * \mathrm{P}<0.01$. critical regulatory roles in AP. It has been reported that miR-29 contributes to apoptosis in AR42J cells by targeting TNFRSF1A[24]; miR-22 and miR-135a promotes the apoptosis of pancreatic acinar cells in acute edematous pancreatitis through ErbB3 and Ptk2[25]; miR-141 plays an important role in the regulation of autophagy in L-arginine-induced acute pancreatitis by targeting HMGB1[11]. In addition, in recent studies, a few up-regulated miRNAs have been reported to be involved in the development and progression of AP. miR-19b up-regulation promotes the necrosis of pancreatic acinar cells, and miR-19b inhibition decreases the rate of pancreatic acinar cell necrosis [26]; miR-21 is up-regulated in a murine model of acute pancreatitis and promotes cellular necrosis by inversely regulating tumor suppressor genes [14]; the expression of miR-216a and miR-216b was considerably increased in a rat model of L-arginine induced acute pancreatitis [27]. However, no studies have focused on the relationship between the low expression of miR-155-5p and AP.

In this research, we built MAP and SAP mouse models to simulate the stages of AP in patients. In the process that brought us to focus on the relative expression of miR-155-5p in peripheral blood, we found that there were 64 miRNAs showing statistically significant differences and among them 34 miRNAs were obviously up-regulated, while 30 miRNAs 
Liu et al.: miR-155-5p Suppresses Acute Pancreatitis Progression by Targeting Rela and Traf3

were down-regulated. However, only the expression of miR-155-5p was the lowest both in MAP and SAP mice. Notably, the expression of miR-155-5p decreased 20-fold in SAP mice (this was the greatest decrease) and approximately 17-fold in MAP mice. For this reason, we selected miR-155-5p as the miRNA to study further in this research, and the results seemed to indicate that miR-155-5p was closely related to the severity of AP. Specifically, we identified and validated two target genes of miR-155-5p, Rela and Traf3, both related to AP, through analysis with the TargetScan software, luciferase gene reporter assays and a series of transfection experiments.

The NF-kB family of transcription factors contains five members, p50, p52, p65 (Rela), Relb and c-Rel $[28,29]$. The NF- $\kappa B$ family of transcription factors plays important roles in a wide range of biological processes such as inflammation, immune responses, cell proliferation, cell differentiation, cell survival and apoptosis [30,31]. Rela is the key subunit of the NF- $\mathrm{kB}$ family [31]. Rela/p65 plays an essential role in the process of mediating NF- $\kappa \mathrm{B}$ signaling and is involved in chondrogenic differentiation, cell survival and catabolic enzyme production [31]. Tumor necrosis factor receptor-associated factor 3 (Traf3) can activate NF$\kappa \mathrm{B}$ through both canonical and non-canonical signaling pathways [32, 33]. Biologic signal transduction mediated by NF- $\mathrm{kB} 1$ is central to inflammation microenvironment changes and

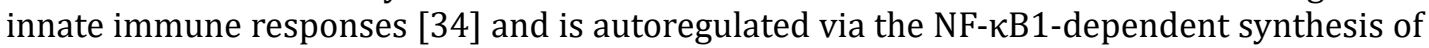
the inhibitor I $\mathrm{\kappa} \mathrm{B} \alpha$. Previous findings demonstrated that Rela and Traf3 were both closely associated with the process of inflammation, which is the main process of AP $[35,36]$.

To further clarify the relation between miRNA-155-5p and AP, we also induced the rat pancreatic cell line AR42J with caerulein or caerulein + LPS to simulate the pathology of MAP or SAP. Thus, we transfected AR42J cells with miR-155-5p mimic, which promoted the expression of miR-155-5p, and clearly found that the target genes Rela, Traf3 and Ptgs2 were all significantly down-regulated at both the transcriptional and post-transcriptional level in the caerulein- and caerulein + LPS-induced group. However, after we transfected the AR42J cells with miR-155-5p inhibitor, which interferes with the expression of miR-155-5p, we found the opposite results. These findings told us that miR-155-5p targeted Rela and Traf3 in AP. When we inhibited the expression of Rela with helenalin in induced AR42J cells, the expression of Traf3 and Ptgs 2 did not significantly change at the transcriptional level, but their proteins were significantly down-regulated at the translational level. In addition, the relative expression of miR-155-5p did not significantly change. The results demonstrated that Traf3 and Ptgs 2 were regulated by Rela, which did not interfere with the expression of miR-155-5p. In order to further prove the relationships among miR-155-5p, Rela, Traf3 and Ptgs2, we collected the pancreatic tissues from control, MAP and SAP mice, and found the inflammatory factors of TNF- $\alpha$ and IL- 6 was highest in SAP mice, followed by in MAP and control mice. The results clarified that the degree of inflammation was serious in SAP mice compared with in MAP mice. In addition, the relative expression of miR-155-5p was lowest in SAP mice but Rela, Traf3 were all higher expression in SAP mice than in MAP and control mice in protein level. These results in vivo illustrated that the expression of miR-155-5p was inversely associated with the degree of the severity of acute pancreatitis, and its targeted genes of Rela and Traf3 were in the regulation of the process. To investigate the expression of miR-155-5p in AP patients, we collected serum samples from 53 AP patients and 53 healthy subjects and found that the expression of miR-155-5p significantly decreased in AP patients. Notably, of the 53 AP patients, 29 were diagnosed with MAP and 24 were diagnosed with SAP. The expression of miR-155-5p was particularly decreased in SAP patients compared with MAP patients. This result further indicated that miR-155-5p was negatively associated with the severity of AP.

In conclusion, through a series of in vivo and in vitro experiments, we found that low expression of miR-155-5p was closely related to the severity of AP and that miR-155-5p regulated pancreatic acinar cell deterioration through the Rela/Traf3/Ptgs 2 signaling pathway. However, in some researches, it has been reported that overexpression of miR155 caused chronic inflammatory state in human [37] and in adipocytes of mice [17]. And there was higher expression of miR-155 in tissues and synovial fibroblasts of patients 


\section{Cellular Physiology Cell Physiol Biochem 2018;51:1584-1599

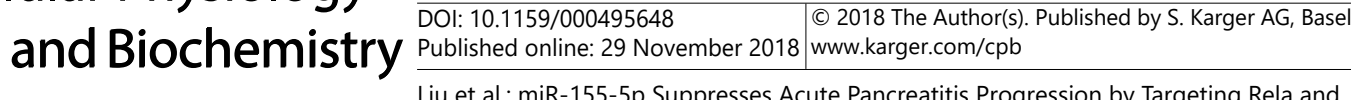 \\ Traf3}

with autoimmune disorders such as rheumatoid arthritis [38]. Actually, in the previous researches, they were focused on the correlation between higher expression of miR-155 and either chronic inflammation or autoimmune disorders in which it needed a relatively long time to maintain this state $[14,22]$.

Our results indicate that miR-155-5p inversely regulated AP development through the Rela/Traf3/Ptgs2 signaling pathway. Since this approach has been successful in treating experimental AP in the mouse, it might become useful also for treating patients with AP. In this research, we were concentrated on the relationship between lower expression of miR-155-5p and acute inflammation in which it needed a relatively short time to form this state. Thus, the expression of miR-155 affect the development of inflammation may be stagedependent, and the exact mechanism needed further research. Actually, we are building the chronic pancreatitis (CP) mice model and we will continue to conduct this research.

\section{Conclusion}

This study discovered a new biomarker of AP: miR-155-5p, which was negatively correlated with the severity of AP and regulated pancreatic acinar cell deterioration through the inhibition of the Rela/Traf3/Ptgs2 signaling pathway.

\section{Acknowledgements}

This work was financially supported by following funds: China Postdoctoral Science Foundation (Grant No. 2017M620347) / Changsha science and technology project (Grant No. kq1706031) / Research Foundation of health commission of Hunan Province (Grant No. C20180423) / Project of scientific research of traditional Chinese medicine in Hunan (Grant No. 201809) /Hunan Provincial People's Hospital Renshu funded (RS201705) / National Natural Science Foundation of China (Grant No. 81701959) / Central guidance of local science and Technology Development Fund (Grant No. 2018CT5008) and Open Research Fund of Hunan Provincial Key Laboratory of Emergency and Critical Care Metabonomics / Hunan Natural Science Foundation (for SL).

\section{Disclosure Statement}

The authors have no conflicts of interest to report.

\section{References}

1 Zaheer A, Singh VK, Qureshi RO, Fishman EK: The revised Atlanta classification for acute pancreatitis: updates in imaging terminology and guidelines. Abdom Imaging 2013;38:125-136.

$>2$ Bollen TL, Singh VK, Maurer R, Repas K, van Es HW, Banks PA, Mortele KJ: A comparative evaluation of radiologic and clinical scoring systems in the early prediction of severity in acute pancreatitis. Am J Gastroenterol 2012;107:612-619.

-3 Busireddy KK, AlObaidy M, Ramalho M, Kalubowila J, Baodong L, Santagostino I, Semelka RC: Pancreatitisimaging approach. World J Gastrointest Pathophysiol 2014;5:252-270.

4 Fabian MR, Sonenberg N: The mechanics of miRNA-mediated gene silencing: a look under the hood of miRISC. Nat Struct Mol Biol 2012;19:586-593.

5 Samir M, Vaas LA, Pessler F: MicroRNAs in the Host Response to Viral Infections of Veterinary Importance. Front Vet Sci 2016;3:86.

6 Liu Z, Wang S, Mi QS, Dong Z: MicroRNAs in Pathogenesis of Acute Kidney Injury. Nephron 2016;134:149153. 


\section{Cellular Physiology and Biochemistry}

7 Alfaradhi MZ, Kusinski LC, Fernandez-Twinn DS, Pantaleao LC, Carr SK, Ferland-McCollough D, Yeo GS, Bushell M, Ozanne SE: Maternal Obesity in Pregnancy Developmentally Programs Adipose Tissue Inflammation in Young, Lean Male Mice Offspring. Endocrinology 2016;157:4246-4256.

8 Kim HY, Kwon HY, Ha Thi HT, Lee HJ, Kim GI, Hahm KB, Hong S: MicroRNA-132 and microRNA-223 control positive feedback circuit by regulating FOXO3a in inflammatory bowel disease. J Gastroenterol Hepatol 2016;31:1727-1735.

-9 Yue J, Song D, Lu W, Lu Y, Zhou W, Tan X, Zhang L, Huang D: Expression Profiles of Inflammation-associated microRNAs in Periapical Lesions and Human Periodontal Ligament Fibroblasts Inflammation. J Endod 2016;42:1773-1778.

10 Rebane A, Akdis CA: MicroRNAs in allergy and asthma. Curr Allergy Asthma Rep 2014;14:424.

11 Zhu H, Huang L, Zhu S, Li X, Li Z, Yu C, Yu X: Regulation of autophagy by systemic admission of microRNA-141 to target HMGB1 in l-arginine-induced acute pancreatitis in vivo. Pancreatology 2016;16:337-346.

12 Gao B, Wang D, Sun W, Meng X, Zhang W, Xue D: Differentially expressed microRNA identification and target gene function analysis in starvation-induced autophagy of AR42J pancreatic acinar cells. Mol Med Rep 2016;14:590-598.

13 Kusnierz-Cabala B, Nowak E, Sporek M, Kowalik A, Kuzniewski M, Enguita FJ, Stepien E: Serum levels of unique miR-551-5p and endothelial-specific miR-126a-5p allow discrimination of patients in the early phase of acute pancreatitis. Pancreatology 2015;15:344-351.

14 Ma X, Conklin DJ, Li F, Dai Z, Hua X, Li Y, Xu-Monette ZY, Young KH, Xiong W, Wysoczynski M, Sithu SD, Srivastava S, Bhatnagar A, Li Y: The oncogenic microRNA miR-21 promotes regulated necrosis in mice. Nat Commun 2015;6:7151.

15 An F, Zhan Q, Xia M, Jiang L, Lu G, Huang M, Guo J, Liu S: From moderately severe to severe hypertriglyceridemia induced acute pancreatitis: circulating miRNAs play role as potential biomarkers. PLoS One 2014;9:e111058.

16 Blenkiron C, Askelund KJ, Shanbhag ST, Chakraborty M, Petrov MS, Delahunt B, Windsor JA, Phillips AR: MicroRNAs in mesenteric lymph and plasma during acute pancreatitis. Ann Surg 2014;260:341-347.

17 Zhang Y, Mei H, Chang X, Chen F, Zhu Y, Han X: Adipocyte-derived microvesicles from obese mice induce M1 macrophage phenotype through secreted miR-155. J Mol Cell Biol 2016;8:505-517.

-18 Soyalp M, Yalcin M, Oter V, Ozgonul A: Investigation of procalcitonin, IL-6, oxidative stress index (OSI) plasma and tissue levels in experimental mild and severe pancreatitis in rats. Bratisl Lek Listy. 2017;118:137-141.

19 Jiang CY, Wang W: Resistin aggravates the expression of proinflammatory cytokines in cerulein-stimulated AR42J pancreatic acinar cells. Mol Med Rep. 2017;15:502-506.

20 Banks PA, Bollen TL, Dervenis C, Gooszen HG, Johnson CD, Sarr MG, Tsiotos GG, Vege SS, Acute Pancreatitis Classification Working Group: Classification of acute pancreatitis--2012: revision of the Atlanta classification and definitions by international consensus. Gut 2013;62:102-111.

-21 Nguyen HV, Gore J, Zhong X, Savant SS, Deitz-McElyea S, Schmidt CM, House MG, Korc M: MicroRNA Expression in a Readily Accessible Common Hepatic Artery Lymph Node Predicts Time to Pancreatic Cancer Recurrence Postresection. J Gastrointest Surg 2016;20:1699-1706.

22 Akamatsu M, Makino N, Ikeda Y, Matsuda A, Ito M, Kakizaki Y, Saito Y, Ishizawa T, Kobayashi T, Furukawa T, Ueno Y: Specific MAPK-Associated MicroRNAs in Serum Differentiate Pancreatic Cancer from Autoimmune Pancreatitis. PLoS One 2016;11:e0158669.

23 Vila-Navarro E, Vila-Casadesus M, Moreira L, Duran-Sanchon S, Sinha R, Gines A, Fernandez-Esparrach G, Miquel R, Cuatrecasas M, Castells A, Lozano JJ, Gironella M: MicroRNAs for Detection of Pancreatic Neoplasia: Biomarker Discovery by Next-generation Sequencing and Validation in 2 Independent Cohorts. Ann Surg 2016; DOI:10.1097/SLA.0000000000001809.

24 Fu Q, Qin T, Chen L, Liu CJ, Zhang X, Wang YZ, Hu MX, Chu HY, Zhang HW: miR-29a up-regulation in AR42J cells contributes to apoptosis via targeting TNFRSF1A gene. World J Gastroenterol 2016;22:4881-4890.

25 Qin T, Fu Q Pan YF, Liu CJ, Wang YZ, Hu MX, Tang Q, Zhang HW: Expressions of miR-22 and miR-135a in acute pancreatitis. J Huazhong Univ Sci Technolog Med Sci 2014;34:225-233.

26 Hu MX, Zhang HW, Fu Q, Qin T, Liu CJ, Wang YZ, Tang Q Chen YX: Functional role of MicroRNA-19b in acinar cell necrosis in acute necrotizing pancreatitis. J Huazhong Univ Sci Technolog Med Sci 2016;36:221-225.

27 Endo K, Weng H, Kito N, Fukushima Y, Iwai N: MiR-216a and miR-216b as markers for acute phased pancreatic injury. Biomed Res 2013;34:179-188.

-28 Dolcet X, Llobet D, Pallares J, Matias-Guiu X: NF-kB in development and progression of human cancer. Virchows Arch 2005;446:475-482.

29 Romanchikova N, Strods A, Strazdina J, Strumfs B, Trapencieris P: A Novel Aziridine-based Bruton's Tyrosine Kinase Inhibitor Induces Apoptosis Through Down-regulation of p65/RelA Phosphorylation on Serine 536 and ERK1/2 in Mantle Cell Lymphoma. Anticancer Res 2016;36:6133-6140.

-30 Li Q Withoff S, Verma IM: Inflammation-associated cancer: NF-kappaB is the lynchpin. Trends Immunol 2005;26:318-325. 


\section{Cellular Physiology and Biochemistry}

Traf3

-31 Kobayashi H, Chang SH, Mori D, Itoh S, Hirata M, Hosaka Y, Taniguchi Y, Okada K, Mori Y, Yano F, Chung UI, Akiyama H, Kawaguchi H, Tanaka S, Saito T: Biphasic regulation of chondrocytes by Rela through induction of anti-apoptotic and catabolic target genes. Nat Commun 2016;7:13336.

-32 He L, Grammer AC, Wu X, Lipsky PE: TRAF3 forms heterotrimers with TRAF2 and modulates its ability to mediate NF-\{kappa\}B activation. J Biol Chem 2004;279:55855-55865.

33 Bista P, Zeng W, Ryan S, Bailly V, Browning JL, Lukashev ME: TRAF3 controls activation of the canonical and alternative NFkappaB by the lymphotoxin beta receptor. J Biol Chem 2010;285:12971-12978.

-34 Locksley RM, Killeen N, Lenardo MJ: The TNF and TNF receptor superfamilies: integrating mammalian biology. Cell 2001;104:487-501.

-35 Wang WY, Chen Y, Su X, Tang D, Ben QW, Yao WY, Chen P, Yuan YZ: Resistin-Like Molecule-alpha Causes Lung Injury in Rats with Acute Pancreatitis by Activating the PI-3K/Akt-NF-kappaB Pathway and Promoting Inflammatory Cytokine Release. Curr Mol Med 2016;16:677-687.

-36 Dur A, Kose H, Kocyigit A, Kocaman 0, Ismayilova M, Sonmez FC: The anti-inflammatory and antioxidant effects of thymoquinone on ceruleine induced acute pancreatitis in rats. Bratisl Lek Listy 2016;117:614618.

-37 O'Connell RM, Rao DS, Baltimore D. microRNA regulation of inflammatory responses. Annu Rev Immunol. 2012;30:295-312.

-38 Faraoni I, Antonetti FR, Cardone J, Bonmassar E: miR-155 gene: a typical multifunctional microRNA. Biochim Biophys Acta 2009;1792:497-505. 